\begin{tabular}{|l|l|} 
REVISTA Revista Educación \\
ISSN: 0379-7082 \\
ISSN: 2215-2644 \\
revedu@gmail.com \\
Universidad de Costa Rica \\
Costa Rica
\end{tabular}

\title{
La lengua indígena en las escuelas ikoots: voces de sus actores
}

\author{
Montero Gutenberg, Gervasio \\ La lengua indígena en las escuelas ikoots: voces de sus actores \\ Revista Educación, vol. 44, núm. 2, 2020 \\ Universidad de Costa Rica, Costa Rica \\ Disponible en: http://www.redalyc.org/articulo.oa?id=44062184038 \\ DOI: https://doi.org/10.15517/revedu.v44i2.40286
}

Esta obra está bajo una Licencia Creative Commons Atribución-NoComercial-SinDerivar 3.0 Internacional 


\section{La lengua indígena en las escuelas ikoots: voces de sus actores}

Indigenous Languages at Ikoots Schools: Stakeholder Voices

Gervasio Montero Gutenberg

DOI: https://doi.org/10.15517/revedu.v44i2.40286

Escuela Normal Bilingüe e Intercultural de Oaxaca, México Redalyc: http://www.redalyc.org/articulo.oa?id=44062184038 monteroikoots@gmail.com

(DD http://orcid.org/0000-0002-3791-4821

Recepción: 25 Febrero 2020

Aprobación: 15 Junio 2020

\section{Resumen:}

En esta colaboración se aborda una experiencia educativa sobre la lengua indígena ombeayiüts'huave' a partir de las voces de sus actores: profesorado y alumnado. La investigación se desarrolla en cuatro escuelas primarias del contexto de los ikoots'huaves', ubicadas en el Istmo de Tehuantepec, Oaxaca, México. El estudio se toca desde la perspectiva de la etnografía escolar, la cual busca mostrar el uso y presencia de la lengua en el ámbito escolar. Los resultados reflejan una heterogeneidad lingüística de la lengua ombeayiüts en la escuela y comunidad. En San Francisco y Santa María del Mar, el proceso de desplazamiento de la lengua lleva a que su presencia en el aula sea nula. En San Dionisio del Mar, la lengua oscila entre ser L1 y L2 en lo comunitario, pero en la escuela tampoco tiene mucha presencia, mientras que, en San Mateo del Mar, se mantiene como L1, lo que la convierte en el medio de comunicación en el aula y en la comunidad. Lo anterior lleva a considerar que la lengua como medio de enseñanza, en tres de las cuatro comunidades: San Francisco, Santa María y San Dionisio, es el español, únicamente en San Mateo, el ombeayiüts se mantiene como medio de comunicación, aunque, como enseñanza es limitada.

Palabras Clave: Lengua ombeayiüts, Educación indígena, Contexto ikoots, Enseñanza.

\section{ABstract:}

The use of Ombeayiüts (also known as 'Huave') at schools, is the subject of this study which also includes recollections of the experiences of teachers and students. Research for this study was conducted at four elementary schools of the Ikoots 'Huaves' located in the Istmo of Tehuantepec, Oaxaca, Mexico. This study was conducted from an ethnographic perspective of the school and aims to demonstrate the use and presence of this language at school. Results reveal that there is linguistic heterogeneity of the ombeayiüts language both at local schools and communities. In San Francisco and Santa María del Mar, indigenous languages have been displaced and are not present at all in the classroom. San Dionisio del Mar's native language ranges between being L1 and L2 in the community. However, this phenomenon is not the case at local schools. In San Mateo del Mar, the native language is still L1 therefore, it is the communication channel in the classroom and in the community. This suggests that teaching is carried out by Spanish in the three communities; San Francisco, Santa María y San Dionisio. Ombeayiüts is only used in San Mateo for communication purposes. Nevertheless, teaching of the language is still limited.

KEYWORDS: Ombeayiüts Language, Indigenous Education, Ikoots Context, Teaching.

\section{INTRODUCCIÓN}

La escuela pensada para los indígenas en el siglo XIX fue limitada en sus alcances (Martín y Escalante, 2017). Aunque hace falta saber con precisión qué pasa con la educación bilingüe o la inserción de las lenguas indígenas en las escuelas, es claro que, como señala Staples (2011), "no hubo consenso en cuanto al papel redentor de la escuela para los indios" (p.353). La realidad educativa que prevalece en el escenario indígena en pleno siglo XXI sigue estando con grandes limitantes y desventajas de atención, programación y cobertura. Por ejemplo, en el estado de Oaxaca, la modalidad educativa con mayor número en preescolar es la general ${ }^{[1]}$ (1987 escuelas) y la indígena (1787 escuelas), en la educación primaria hay más de 3000 escuelas generales, pero sólo 1753 indígenas. 
La educación indígena en México se ve materializada en 1938 con la creación de la Dirección General de Educación Indígena (DGEI), después de haber pasado por diversas políticas lingüísticas para incorporar al indígena a la sociedad nacional a través de la imposición del español en forma directa o a través de la enseñanza bilingüe, o como en 1917 cuando se propuso una aculturación a nivel nacional (Brice, 1986), pues "desde la Conquista a la fecha, la estructura política y las instituciones educativas estatales han impulsado la aculturación de estos pueblos indígenas, imponiendo la lengua y la cultura de la sociedad, primero colonia española, posteriormente mexicana" (Hamel y Muñoz, 1981, p.127).

Con la creación de la DGEI se lograba un objetivo primordial relacionado a la elaboración de programas, metodologías y capacitación profesional específica para la atención de la niñez indígena (DGEI, 2004). Sin embargo, "en el fondo de lo que se trataba era de definir un modelo para la incorporar a los indígenas a la nación” (Zolla y Zolla, 2004, p. 245), no obstante, también surgen paradigmas que en teoría abonan al uso de la lengua en la enseñanza, como lo es la educación bilingüe, educación bilingüe bicultural y educación bilingüe intercultural (Muñoz, 2011), aunque siguen siendo paradigmas educativos, alejadas de la realidad y de la praxis.

Por tal motivo, en este artículo se aborda el problema de la educación indígena en el contexto de la cultura ikoots, específicamente en cuanto al uso y presencia de la lengua ombeayiüts en el escenario escolar y comunitario. En un primer momento, se aportan reflexiones generales sobre las lenguas originarias y la educación indígena. Después, se enfatiza la perspectiva etnográfica y el etnográfico interpretativo como base metodológica que guiaron el trabajo. Posteriormente, se adentra en el contexto de la cultura ikoots y de la lengua ombeayiüts para resaltar el escenario de las escuelas a través de sus principales características, enfatizando el estatus de la lengua originaria, la heterogeneidad lingüística, la enseñanza de la lengua y se cierra con las reflexiones finales.

En este marco y dado que el objeto de la investigación es mostrar el uso y presencia de la lengua en el contexto escolar indígena y a la vez, enfatizar el contraste con la situación sociolingüística del contexto comunitario ikoots ${ }^{[2]}$ de la región del istmo de Tehuantepec, México se seleccionó una escuela primaria por cada comunidad: Moisés Sáenz en San Mateo del Mar (SMo), Benito Juárez en San Dionisio del Mar (SDo), Doce de Octubre en San Francisco del Mar (SFo) y Lázaro Cárdenas en Santa María del Mar (SMa); las tres primeras pertenecen a la modalidad de educación indígena y la última a la general. Sin embargo, debido que en la comunidad de SMa no existe primaria bilingüe, se trabajó con la escuela del sistema general para así poder compararla con las otras comunidades.

Se trata de escenarios donde existe una diferenciada presencia y uso de la lengua ombeayiüts en el ámbito comunitario y, sobre todo, en el escolar.

\section{LAS LENGUAS ORIGINARIAS Y LA EDUCACIÓN INDÍGENA}

En el contexto mexicano, el reconocimiento de lo que se ha considerado la educación indígena se ha dado de manera formal, existen políticas y programas educativos destinados a ella. Enmarcada en un enfoque intercultural desde el siglo XX, se concreta con la creación de la Coordinación General de Educación Intercultural y Bilingüe (CGEIB) en 1998, con "la idea de que la interculturalidad puede ser una posibilidad de superar relaciones asimétricas de poder entre culturas con nuevas relaciones basadas en la igualdad y el respeto" (Sandoval, 2004, p. 57). Sin embargo, esto sigue alejado de la realidad de las poblaciones indígenas, ya que, a veces la interculturalidad se visualiza desde lo relacional o funcional, como mecanismos asistencialistas donde el objetivo final sigue siendo la integración o inclusión de las lenguas y culturas indígenas a la vida nacional (Walsh, 2009, Tubino, 2005). Lejos de establecer una nueva relación entre los estados y las comunidades indígenas, la tendencia es un proceso de oficialización y retorización de la interculturalidad (Sartorello, 2009). 
Por ello, los avances existentes en educación indígena son limitados, por ejemplo, en 2016-2017, la producción de libros en lenguas indígenas sólo abarcó 38 de las 68 lenguas indígenas, es decir, no se cubrió a $45 \%$ de las lenguas, la enseñanza de lenguas originarias prevalece únicamente como asignatura, prevalece la carencia de metodologías pedagógicas específicas para la enseñanza de lenguas originarias (como primeras o segundas lenguas) al igual que del español, escasos avances en el proceso de normalización de las lenguas indígenas (Instituto Nacional para la Evaluación de la Educación, 2017).

Asimismo, investigaciones efectuadas en escuelas de educación indígena (Montero, 2012; Czarny, 2008; Saldívar, 2006) señalan que no se trabaja con las lenguas indígenas por diferentes razones como: el desplazamiento lingüístico, el estatus del español como primera lengua, hablantes pasivos de la lengua indígena (tanto profesorado como alumnado), la desubicación lingüística ${ }^{[3]}$ del profesorado. Asimismo, como señala el INEE (2017), alrededor de la mitad de las y los docentes de escuelas de modalidad indígena no habla una lengua indígena ( $47 \%$ en preescolar y 53\% en primaria), que afianza lo que afirma Barriga (2018) que "el bilingüismo, es sólo un término sin contenido real que, si acaso se lograra, es asimétrico y en franca transición hacia la supremacía del español” (p. 50).

Por tal razón, es importante reflexionar sobre la diversidad sociolingüística que caracteriza a las escuelas de educación indígena en Oaxaca, en específico del contexto de los ikoots. Este trabajo abona en mostrar la relación entre la enseñanza como paradigma educativo y lingüístico, y la escuela como espacio de encuentro o desencuentro lingüístico y cultural.

A pesar del discurso de una educación indígena en el sistema educativo, la educación que se imparte en las escuelas en muchos casos sigue siendo esencialmente monolingüe en español y el uso de las lenguas indígenas sólo se da de manera marginal o como puente, encaminado hacia un bilingüismo sustractivo, donde prevalece la homogeneización cultural (Skutnabb y Dunbar, 2010). Se puede decir entonces que las lenguas indígenas en México han sido tratadas por el Estado como un instrumento asimilacionista, conduciendo al proceso de aculturación y pérdida identitaria. Desde la época colonial, la trayectoria de la política lingüística del Estado se proyecta en ampliar el español a todo el territorio nacional, buscando eliminar las lenguas y culturas indígenas, ya que, en realidad las políticas educativas han contribuido a acelerar el desuso y el abandono de las lenguas originarias (Bensasson, 2010).

Por ello, en educación indígena el panorama es sumamente complejo, pues según el propio profesorado bilingüe, en las escuelas, la enseñanza que se ofrece empieza a ser mayoritariamente monolingüe en español, esta también debido a factores como: el desinterés del propio alumnado, la negativa de los padres hacia el aprendizaje de la lengua indígena, la preferencia del inglés sobre la lengua originaria y aunado a lo que se ha mencionado, el profesorado que labora en el medio indígena, desconocen o ya no hablan la lengua o, en el mejor de los casos, la hablan, pero no la saben leer ni escribir.

Por tal razón, existen escenarios donde el proceso de enseñanza y aprendizaje se desarrolla sólo en español y únicamente de manera excepcional se utilizan las lenguas indígenas, ello hace que, "el peligro de la interculturalidad puede disfrazar la unilateralidad al proponer una política indígena desde el mestizo, porque está pensada únicamente como necesidad de las comunidades indígenas y no de la comunidad nacional” (Barriga, 2018, pp. 94-95). Hace falta elementos que pueda visualizar de otra forma la educación indígena (Gasché, 2008), por lo que profundizar sobre la presencia y uso de la lengua resulta relevante (Meyer y Hamel, 2009).

\subsection{Perspectiva metodológica}

El marco de esta investigación se desarrolla desde una perspectiva de la etnografía de la práctica escolar (González, 2013; Rockwell y González, 2012; Bertely 2000), donde entran en juego: el encuentro profesorado-alumnado-conocimiento en situaciones sociointeraccionales, las relaciones contraídas por los agentes de la institución escolar y los factores socioculturales y lingüísticos que afectan la dinámica escolar. Se complementa con el método etnográfico interpretativo, ya que "documenta lo no documentado de la realidad social” (Rockwell, 2009, p. 21). Ambas miradas se interrelacionan para exponer una realidad en 
específico, como es el uso y presencia de la lengua en los centros escolares y en las aulas, porque, a partir del trabajo descriptivo y teóricamente orientado de la realidad, necesita documentarse para luego interpretarse (Rockwell, 2009; Serra, 2004). Para ello, la entrevista ${ }^{[4]}$ semiestructurada permitió registrar la experiencia de enseñanza y aprendizaje de la lengua ombeayiüts (profesorado y alumnado) y las percepciones que tienen acerca de ella (Brenner, 2006).

TABLA 1

Perfil del profesorado entrevistado

\begin{tabular}{|c|c|c|c|c|c|c|c|c|}
\hline \multirow{3}{*}{$\begin{array}{l}\text { Referen- } \\
\text { cia }^{[5]}\end{array}$} & \multirow[t]{3}{*}{$\begin{array}{l}\text { Comu- } \\
\text { nidad }\end{array}$} & \multirow[t]{3}{*}{ Edad } & \multirow[t]{3}{*}{ Escuela } & \multirow{3}{*}{$\begin{array}{l}\text { Grado } \\
\text { que } \\
\text { atiende }\end{array}$} & \multirow[t]{3}{*}{\begin{tabular}{|l}
$\begin{array}{l}\text { Nivel de } \\
\text { estudio }\end{array}$ \\
\end{tabular}} & \multicolumn{3}{|c|}{$\begin{array}{l}\text { Habla } \\
\text { ombeayiüts }\end{array}$} \\
\hline & & & & & & Sí & & No \\
\hline & & & & & & L1 & L2 & \\
\hline P1 & SFO & 50 & $\begin{array}{l}\text { Prim. Bil. } 12 \\
\text { de octubre }\end{array}$ & Bro B & $\begin{array}{l}\text { Normal } \\
\text { Superior } \\
\text { (Pasante) }\end{array}$ & & & $\mathrm{x}$ \\
\hline P2 & SFO & 32 & $\begin{array}{l}\text { Prim. Bil. } 12 \\
\text { de octubre. }\end{array}$ & 1 ro $B$ & $\begin{array}{l}\text { Maestría } \\
\text { (pasante). }\end{array}$ & & & $\mathrm{x}$ \\
\hline P3 & SDo & 43 & $\begin{array}{l}\text { Prim. Bil. } \\
\text { Benito Juárez }\end{array}$ & 2do. A & UPN (Pasante) & & $\mathrm{x}$ & \\
\hline P4 & SDo & 45 & $\begin{array}{l}\text { Prim. Bil. } \\
\text { Benito Juárez }\end{array}$ & 5to A & $\begin{array}{l}\text { Normal } \\
\text { Superior } \\
\text { (pasante) }\end{array}$ & $\mathrm{x}$ & & \\
\hline P5 & SMa & 35 & $\begin{array}{l}\text { Prim. General } \\
\text { "Lázaro } \\
\text { Cárdenas" }\end{array}$ & 3ro & $\begin{array}{l}\text { UPN (4 } \\
\text { semestre) }\end{array}$ & & & $\mathrm{x}$ \\
\hline P6 & SMa & 40 & $\begin{array}{l}\text { Prim. General } \\
\text { "Lázaro } \\
\text { Cárdenas" }\end{array}$ & 4to & $\begin{array}{l}\text { Normal } \\
\text { titulado }\end{array}$ & & & $\mathrm{x}$ \\
\hline P7 & SMo & 56 & $\begin{array}{l}\text { Prim. Bil. } \\
\text { Moisés Sáenz }\end{array}$ & $1 \mathrm{ro} A$ & $\begin{array}{l}\text { Lic. Pedagogía } \\
\text { (titulado) }\end{array}$ & $\mathrm{x}$ & & \\
\hline P8 & SMo & 29 & $\begin{array}{l}\text { Prim. Bil. } \\
\text { Moisés Sáenz }\end{array}$ & 6to $\mathrm{A}$ & UPN (titulada) & $\mathrm{x}$ & & \\
\hline
\end{tabular}

Fuente: Elaboración propia

Se entrevistó dos integrantes del profesorado de cada escuela con diferentes perfiles como se menciona en la Tabla 1, estos fueron designados de forma aleatoria por los directores de la institución, pues el fin era conocer su percepción y experiencia con el trabajo de la lengua. Así también, para conocer la percepción del alumnado con respecto a la presencia del ombeayiüts en el aula, se entrevistó a 8 elementos de sexto grado, dos de cada una de las escuelas de estudio con diferentes perfiles (ver Tabla 2). Se optó por el alumnado de sexto grado por la edad y experiencia escolar que pudieran tener con la lengua en el aula y en la escuela. 
TABLA 2

Perfil del alumnado

\begin{tabular}{|c|c|c|c|c|c|c|c|c|}
\hline \multirow[t]{2}{*}{$\begin{array}{l}\text { Referen } \\
\text {-cia }\end{array}$} & \multirow[t]{2}{*}{ Nombre } & \multirow[t]{2}{*}{ Comuni- dad } & \multirow[t]{2}{*}{ Edad } & \multirow[t]{2}{*}{ Escuela } & \multirow{2}{*}{$\begin{array}{l}\text { Grado } \\
\text { que } \\
\text { cursa }\end{array}$} & \multicolumn{3}{|c|}{$\begin{array}{l}\text { Habla } \\
\text { ombeayiüts }\end{array}$} \\
\hline & & & & & & & L2 & No \\
\hline$A 1^{[6]}$ & $\begin{array}{l}\text { Alumna } \\
1\end{array}$ & SMo & 11 & $\begin{array}{l}\text { Prim. Bil. } \\
\text { Moisés Sáenz. }\end{array}$ & $6 \mathrm{~A}$ & $\mathrm{x}$ & & \\
\hline A2 & $\begin{array}{l}\text { Alumno } \\
2\end{array}$ & SMo & 12 & $\begin{array}{l}\text { Prim. Bil. } \\
\text { Moisés Sáenz. }\end{array}$ & $6 \mathrm{~A}$ & $\mathrm{x}$ & & \\
\hline $\mathrm{A} 3$ & $\begin{array}{l}\text { Alumna } \\
3\end{array}$ & SDo & 11 & $\begin{array}{l}\text { Prim. Bil. } \\
\text { Benito Juárez }\end{array}$ & $6 \mathrm{~A}$ & & $\mathrm{x}$ & \\
\hline A4 & $\begin{array}{l}\text { Alumno } \\
4\end{array}$ & SDo & 11 & $\begin{array}{l}\text { Prim. Bil. } \\
\text { Benito Juárez }\end{array}$ & $6 \mathrm{~A}$ & & & $\mathrm{x}$ \\
\hline A5 & $\begin{array}{l}\text { Alumna } \\
5\end{array}$ & SMa & 11 & $\begin{array}{l}\text { Prim. General } \\
\text { "Lázaro } \\
\text { Cárdenas" }\end{array}$ & $6 \mathrm{~A}$ & & & $\mathrm{x}$ \\
\hline A6 & $\begin{array}{l}\text { Alumna } \\
6\end{array}$ & SMa & 10 & $\begin{array}{l}\text { Prim. Rural } \\
\text { "Lázaro } \\
\text { Cárdenas" }\end{array}$ & $6 \mathrm{~A}$ & & & $\mathrm{x}$ \\
\hline $\mathrm{A} 7$ & $\begin{array}{l}\text { Alumno } \\
7\end{array}$ & SFO & 11 & $\begin{array}{l}\text { Prim. Bil. "12 } \\
\text { de octubre" }\end{array}$ & $6 \mathrm{~A}$ & & & $\mathrm{x}$ \\
\hline A8 & $\begin{array}{l}\text { Alumna } \\
8\end{array}$ & SFO & 10 & $\begin{array}{l}\text { Prim. Bil. "12 } \\
\text { de octubre" }\end{array}$ & $6 \mathrm{~A}$ & & & $\mathrm{x}$ \\
\hline
\end{tabular}

Fuente: Elaboración propia

A partir de los datos investigados, se puede conocer las conexiones entre las condiciones sociolingüísticas comunitarias y el papel de la lengua indígena en la escuela, y entre su enseñanza o no dentro del aula y la concepción sobre su uso por los agentes implicados en el proceso educativo.

\section{EL CONTEXTO DE LA CULTURA IKOOTS Y DE LA LENGUA OMBEAYIÜTS}

La lengua ombeayiüts es una de las 16 lenguas originarias habladas en el estado de Oaxaca, principalmente en SFo, SMo, SDo y SMa. La situación sociolingüística de la lengua es diferente en cada una de las comunidades, como se menciona la tabla 3 .

TABLA 3

Porcentaje de hablantes de ombeayiüts por comunidad

\begin{tabular}{|l|l|l|l|l|}
\hline COMUNIDAD & $\begin{array}{l}\text { CENSO 2000 } \\
\%\end{array}$ & $\begin{array}{l}\text { CONTEO 2005 } \\
\%\end{array}$ & $\begin{array}{l}\text { CENSO 2010 } \\
\%\end{array}$ & CONTEO 2015\% \\
\hline SMO & 85.5 & 86.4 & 86.6 & 97.6 \\
\hline SDO & 53.4 & 51.0 & 47.0 & 46.7 \\
SMa & 18.9 & 16.2 & 17.3 & 16.9 \\
\hline SFO & 18.8 & 13.4 & 14.6 & 13.6 \\
\hline
\end{tabular}

Fuente: Elaboración propia con base en datos del Instituto Nacional de Estadística y Geografía [INEGI] (2000, 2005, 2010, 2015).

Como muestra la tabla, tres comunidades tienen un decrecimiento en el porcentaje de hablantes. Si se considera el censo de 2000 y el conteo intercensal de 2015: SMa con 2\%, SFo con 5.2\% y SDo con un 6.7\% respectivamente. A diferencia de SMo que tuvo un crecimiento de $12.1 \%$, lo cual indica que hay vitalidad de la lengua. Se visualiza entonces que tres comunidades llevan tendencia a procesos de desplazamiento acelerados, 
como es el caso de SFo y SMa mayormente y las repercusiones se reflejan de forma directa en la vivencia en el espacio escolar. Al respecto García (2017) documenta:

\begin{abstract}
Mientras en San Mateo el huave es la lengua que predomina en la vida cotidiana de todas las generaciones, en San Dionisio únicamente los adultos mayores son bilingües en huave y español, mientras los adultos jóvenes tienden a comunicarse en español y los jóvenes muestran una casi nula competencia en el manejo de la lengua vernácula. San Francisco del Mar es el municipio donde el huave está más amenazado: el español constituye la lengua del dominio común y los hablantes bilingües de huave y español se encuentran únicamente entre la población mayor de sesenta años. (p.26)
\end{abstract}

\title{
3.1 Las escuelas ikoots
}

El escenario de las escuelas ikoots es muy peculiar, que a lo mejor no distan de la vivencia cotidiana de otros contextos, sin embargo, tienen matices muy específicos que los hacen únicos. La presencia que tiene la enseñanza y aprendizaje de la lengua, las metodologías utilizadas y las problemáticas que enfrenta el profesorado y alumnado en este proceso son distintas también. Las escuelas ikoots son espacios donde se configuran acciones, encuentros, desencuentros, pero que mantienen una historia como dice Tallè (2015):

La historia de las relaciones entre los huaves o ikoots de San Mateo del Mar y la escuela es semejante a la de muchas otras comunidades indígenas de México y del continente americano entero. Es una historia de imposición y resistencia, de desconfianza y renuencia y, recientemente, de apropiación y reivindicación. (p.160)

Justo en esta reivindicación en que cada una de las escuelas se configuran en sus contextos definidos de acuerdo a la presencia o no de la lengua. Asimismo, las características de las escuelas de estudio reflejan a la vez la situación sociolingüística de la comunidad.

TABLA 4

Total de escuelas (primaria y preescolar) en el contexto ikoots

\begin{tabular}{|l|l|l|l|l|}
\hline $\begin{array}{l}\text { Subsistema } \\
\text { Comunidad }\end{array}$ & $\begin{array}{l}\text { Primaria } \\
\text { Indigena }\end{array}$ & $\begin{array}{l}\text { Primaria } \\
\text { General }\end{array}$ & $\begin{array}{l}\text { Preescolar } \\
\text { Indigena }\end{array}$ & $\begin{array}{l}\text { Preescolar } \\
\text { General }\end{array}$ \\
\hline San Mateo del Mar & 15 & 1 & 15 & 0 \\
\hline San Dionisio del Mar & 5 & 4 & 5 & 0 \\
San Francisco del Mar & 6 & 6 & 8 & 1 \\
Santa María del Mar & 0 & 1 & 1 & 0 \\
\hline
\end{tabular}

Fuente: Elaboración propia.

Como aparece en la Tabla 4, en SMo prevalecen mayoritariamente las escuelas del subsistema de educación indígena tanto en primaria ( 15 escuelas, de las cuales 4 pertenecen a la modalidad de educación primaria comunitaria indígena del Consejo Nacional de Fomento Educativo (CONAFE)) como en preescolar (15, de las cuales 3 son del CONAFE), mientras que en las otras tres comunidades la situación es distinta, existe la presencia casi del mismo porcentaje de ambas modalidades educativas, lo que lleva a pensar que en las escuelas del sistema general no se visibiliza la lengua indigena.

Por ello, las escuelas ikoots reflejan y presentan diferentes características y situaciones lingüísticas.

\subsubsection{Escuela Primaria Bilingüe "12 de octubre". SFo}

Esta institución está ubicada en la cabecera municipal de SFo. Es de organización completa ${ }^{[7]}$. El cuerpo docente de la institución consta de un total de 14 frente a grupo: 4 mujeres y 10 hombres. La mayoría son de la variante de SFo y 4 son de SDo. El profesorado de SFo tienen como L1 al español, mientras que los de SDo únicamente una profesora dijo entenderla y hablarla. 
La escuela tiene una matrícula de 280 estudiantes, 136 niñas y 144 niños. El alumnado no habla el ombeayiüts, sino el español. El director de la institución comenta: "aquí ya no hablamos el idioma, algunos profesores solo entendemos pequeñas palabras, el alumnado llega hablando el español, es lo que aprenden en sus casas" (P2, comunicación personal, 26 septiembre 2018). Esta cuestión se ve reflejada en el recreo, el alumnado juega, platica, conversa siempre en español.

El director declara que, a pesar de la situación, hay intenciones de trabajar la lengua, lo que ha sido relevante desde la perspectiva de las y los habitantes, como lo expresa una madre de familia:

Mando mi hijo a la 12 (12 de octubre), porque ahí enseñan el idioma. Quiero que él aprenda, aunque sea un poco, en cambio en las otras escuelas no enseñan nada de eso, porque los maestros no son de aquí. Así él nos enseñará después (risas) porque su papá y yo, no hablamos nada. (Y. Vargas, comunicación personal, 25 septiembre 2018)

Esta afirmación cobra relevancia porque en la cabecera municipal de SFo (ver tabla 4), existen otras tres escuelas primarias, una bilingüe "Once de enero" bidocente y otras dos del sistema general. Pero el hecho de "enseñar" la lengua ombeayiüts es un criterio de selección para madres y padres, aunque dicha enseñanza se limita a algunas palabras aisladas, pero ha hecho considerar a la escuela, como uno de los pocos contextos de presencia de la lengua.

En toda la institución la lengua de comunicación predominante es el español, porque es la L1 tanto del profesorado como del alumnado en correspondencia con el $13.6 \%$ de la población general hablante de la lengua (INEGI, 2015), es decir, la presencia comunitaria de la lengua es limitada.

\subsubsection{Escuela Primaria Bilingüe "Benito Juárez". SDo}

Esta institución se encuentra en SDo, Pueblo Nuevo. Es de organización completa y cuenta con seis integrantes del cuerpo docente frente a grupo y un director. Seis son de SDo y hablantes del ombeayiüts, tres lo tienen como L1, dos como L2 y una profesora es moel ${ }^{[8]}$, hablante de la lengua náhuatl.

Tiene un total de 122 personas alumnas, 68 niños y 54 niñas. La mayoría del alumnado afirman que no hablan la lengua ombeayiüts y esto es visible en los espacios escolares donde el español es la lengua predominante como medio de comunicación e interacción en todo momento. Esto lo confirma el director de la escuela:

Aquí en la escuela, los niños ya casi no hablan el huave, aunque no hemos hecho un diagnóstico puntual en cada uno de los grados en este inicio del ciclo escolar, pero hay niños que le entienden, aunque no lo hablen y otros que sí le entienden y lo hablan, aunque no son muchos, pero los hay. (P1, comunicación personal, 14 septiembre 2018)

La tendencia señala que el español se ha posicionado en muchos espacios y se está convirtiendo en L1. La transmisión y adquisición del ombeayiüts como L1 también es limitada.

\subsubsection{Escuela Primaria General "Lázaro Cárdenas". SMa}

Esta escuela pertenece al sistema general, es de organización completa. La totalidad del profesorado son de fuera, hablantes del zapoteco. La comunidad es una agencia municipal perteneciente al municipio de Juchitán de Zaragoza (hablantes de la lengua zapoteco del istmo). En la localidad, la lengua ombeayiüts aún tiene presencia como L1 pero se concentra en la generación adulta. El español también ya predomina como L1, como declara el director de la escuela:

Los niños de esta escuela no hablan el dialecto ( $\mathrm{sic}$ ), todos se comunican en español y esto se explica porque, la lengua predominante en la comunidad es el español, las nuevas generaciones ya no hablan el dialecto, solamente los abuelos, por eso, nadie de los niños que tenemos en la escuela habla la lengua, sino que todos hablan el español, porque es la lengua que sus papás les enseñaron en la casa. (P5, comunicación personal, 22 septiembre 2018). 
Esta afirmación se visualiza en diversos espacios de la escuela: en el aula, los sanitarios, los puestos de venta, la dirección de la escuela, el campo deportivo y en la plaza cívica, donde el medio de comunicación del alumnado es a través del español. Otros niños y niñas confirmaron, no ser hablantes del ombeayiüts.

Como se ha descrito, en la comunidad y en el ámbito escolar se empieza a consolidar el español como L1.

\subsubsection{Escuela Primaria Bilingüe "Moisés Sáenz". SMo}

Esta institución se encuentra ubicada en Barrio Nuevo, SMo. Es de organización completa, integrada por 12 profesorado frente a grupo (dos grupos por cada grado) y un director. Nueve integrantes de la planta docente son de SMo, hablantes del ombeayiüts como L1, una de SDo, una de SMa (hablantes del ombeayiüts como L2) y dos hablantes del zapoteco, de Ixtepec y de San Blas Atempa.

La escuela tiene un total de 305 personas alumnas. La mayoría habla el ombeayiüts como L1, y esto se ve reflejado en los diferentes espacios escolares, el alumnado se comunica en su L1: al jugar en el recreo, al comprar, al acercarse al profesorado, el medio de comunicación e interacción es el ombeayiüts, incluso, en las actividades de educación física, aunque el o la docente les hable en español, el alumnado responde en ombeayiüts. Esta situación tiene relación con los datos del INEGI (2015), donde un 97.6\% son hablantes de la lengua. El director de la escuela comenta al respecto:

En nuestra escuela, nuestros alumnos hablan todavía nuestra lengua y como la mayoría de los profesores son de aquí y hablan también la lengua, entonces eso ha sido una gran ventaja, porque algunos niños no hablan para nada el español solo ombeayiüts ..., esto refleja que en la comunidad se habla todavía el ombeayiüts y eso es bueno. (P6, comunicación personal, 08 octubre 2018).

Por lo que, la tendencia que se presenta es que, el ombeayiüts es la L1 y el español es la L2 tanto en la escuela como en la comunidad.

En resumen, existe perfiles sociolingüísticos diversos en las escuelas ikoots como se sintetiza en la Tabla 5.

TABLA 5

Perfiles sociolingüísticos en las escuelas ikoots

\begin{tabular}{|c|c|c|c|c|c|c|}
\hline \multirow{2}{*}{$\begin{array}{l}\text { Comu- } \\
\text { nidad }\end{array}$} & \multirow[t]{2}{*}{ Escuela } & \multirow[t]{2}{*}{ Modalidad } & \multicolumn{4}{|c|}{ Perfil lingüístico declarado } \\
\hline & & & Alumnado & Profesorado & $\begin{array}{l}\text { Variante del } \\
\text { profesorado }\end{array}$ & $\begin{array}{l}\text { Comunidad } \\
\text { (INEGI, } \\
2015 \text { ) } \\
\text { Hablantes } \\
\%\end{array}$ \\
\hline SFo & $\begin{array}{l}12 \text { de } \\
\text { Octubre }\end{array}$ & $\begin{array}{l}\text { Primaria } \\
\text { bilingüe }\end{array}$ & Español L1 & $\begin{array}{l}\text { Español L1 } \\
\text { Ombeayiüts } \\
\text { L2 }\end{array}$ & SFO SDO & $\begin{array}{l}13.6 \% \\
\text { Español L1 }\end{array}$ \\
\hline SDo & $\begin{array}{l}\text { Benito } \\
\text { Juárez }\end{array}$ & $\begin{array}{l}\text { Primaria } \\
\text { bilingüe }\end{array}$ & $\begin{array}{l}\text { Español L1 } \\
\text { Ombeayiüts } \\
\text { L2 }\end{array}$ & $\begin{array}{l}\text { Ombeayiüts } \\
\text { L1 }\end{array}$ & SDo & $\begin{array}{l}46.7 \% \\
\text { Español L1 } \\
\text { Ombeayiüts } \\
\text { L1-L2 }\end{array}$ \\
\hline SMa & $\begin{array}{l}\text { Lázaro } \\
\text { Cárdenas }\end{array}$ & $\begin{array}{l}\text { Primaria } \\
\text { general }\end{array}$ & Español L1 & $\begin{array}{l}\text { Español L1 } \\
\text { Zapoteco L1 }\end{array}$ & Juchitán & $\begin{array}{l}16.9 \% \\
\text { Español L1 }\end{array}$ \\
\hline SMo & $\begin{array}{l}\text { Moisés } \\
\text { Sáenz }\end{array}$ & $\begin{array}{l}\text { Primaria } \\
\text { bilingüe }\end{array}$ & $\begin{array}{l}\text { Ombea-yiüts } \\
\text { L1 }\end{array}$ & $\begin{array}{l}\text { Ombea-yiüts } \\
\text { L1 } \\
\text { Ombea-yiüts } \\
\text { L2 }\end{array}$ & $\begin{array}{l}\text { SMo SMa } \\
\text { SDo }\end{array}$ & $\begin{array}{l}97.6 \% \\
\text { Ombeayiüts } \\
\text { L1 Español } \\
\text { L2 }\end{array}$ \\
\hline
\end{tabular}

Fuente: Elaboración propia. 
Las características que se han señalado sobre la situación de la lengua en cada una de las comunidades y escuelas cobran relevancia sobre todo en las implicaciones metodológicas, es decir, cómo se aborda o se enseña en el aula. De acuerdo con la UNICEF (2018), en México el 9.5\% de las escuelas preescolares indígenas y 8.3\% de las primarias indígenas, el profesorado no habla las lenguas maternas de la comunidad y, por consiguiente, no enseñan en la lengua del alumnado.

\section{Heterogeneidad LINGÜístiCA Y ENSEÑANZA DE LA LENGUA}

Como se ha mencionado, el perfil lingüístico del alumnado es muy diferenciado en las diversas escuelas. En SDo uno de los entrevistados afirma:

No es común que tengamos en el aula alumnos que hablen el umbeyajts, aunque en ocasiones no nos damos cuenta, a veces también los padres ya no les enseñan en la casa, aunque algunos papás lo hablan todavía o sus abuelos. Pero este ciclo si tengo una niña que habla bien el idioma, creo que "aprendió” primero el español y luego umbeyajts, pero ella es la que podría apoyarme en la clase para que ayude a sus compañeros a aprenderlo. (P4, comunicación personal, 13 octubre 2018).

En SDo aún hay presencia de la lengua, pero esta empieza a tener el estatus de L2, como en el caso anterior que se cita. Una de las alumnas entrevistadas comenta sobre su adquisición y aprendizaje de la lengua originaria:

Hablo el español, es la lengua que me enseñaron primero en la casa. Pero también hablo el umbeyajts más o menos (risas), eso lo aprendí ya grande, mis papás y mis abuelos lo hablan muy bien. Mis papás me enseñaron, yo escuchaba sus pláticas e iba aprendiendo también poco a poco, también me hablan en umbeyajts para hacer algún trabajo en la casa. Mi abuelita también me enseña y ella si me habla todo el tiempo en umbeyajts. Aquí en la escuela fui aprendiendo un poco más también... (A3, comunicación personal, 14 septiembre 2018)

Por otro lado, se encuentran SFo y SMa. El profesorado es consciente de la realidad sociolingüística de la lengua en la comunidad, asumiendo en primera instancia que ellos no hablan la lengua, y afirman que el alumnado llega a la escuela con el español como L1.

Tengo un total de 24 niños de los cuales nadie habla el umbeyajts, su primera lengua es el español. Los padres de familia tampoco lo hablan, porque he realizado reuniones donde les he preguntado y todos han comentado que solamente hablan el español. Por eso todos los niños llegan a la escuela hablando esta lengua y en ella nos comunicamos. (P2, comunicación personal, 18 octubre, 2018).

En la misma situación se encuentra SMa. Uno de los entrevistados asevera que la lengua ya no tiene presencia en la comunidad, sobre todo en las nuevas generaciones, y que madres y padres no quieren que se enseñe la lengua.

En contraparte, en SMo una integrante del profesorado opina que es muy importante trabajar el ombeayiüts en el aula, aparte de ser la L1 del alumnado, considera que también favorece en el aprendizaje:

Considero que el papel de la lengua en la escuela es muy importante, debemos de enseñarla desde los primeros grados, porque, aunque el alumnado habla muy bien, pero nosotros aquí en la escuela debemos de retomarla, porque eso ayudará a que los niños puedan aprender mejor o les ayudará en su aprendizaje. Por eso para mí, la lengua es primordial en la enseñanza. (P7, comunicación personal, 08 noviembre 2018)

Esta actitud hacia la lengua se ve manifestada también en la percepción del alumnado de esta comunidad:

No me gusta aprenderlo ni hablarlo para nada, a parte es muy difícil. No sé porque, pero no me llama la atención, prefiero el español o el inglés, creo que me van a servir más y ayudar para seguir estudiando, porque el huave no tiene mucho reconocimiento, solo se habla un poco aquí en la comunidad y no se habla en muchas partes. Por eso pienso que no me servirá de mucho, mejor aprendo otras cosas, mi papá me dice que aprenda a leer y escribir bien el español porque no tengo tan buena ortografía y me regañan por eso. (A5, comunicación personal, 22 septiembre 2018) 
En contraste, las declaraciones del alumnado de las otras tres comunidades son diferentes. Consideran que la lengua ombeayiüts es parte fundamental de su propia identidad como ikoots. La lengua sigue siendo un elemento identitario, un pasaporte de pertenencia a la comunidad, como se lee en el siguiente comentario:

Pienso que el ombeayiüts es muy importante, me ayuda a mostrarme que soy ikoots, que soy de este pueblo, que vivo aquí, de lo contario la gente me diría que estoy mal, que hago mal al hablar español estando aquí, me dirían muchas cosas o me rechazarían y tienen razón. Porque aquí la gente habla ombeayiüts y no estaría bien que yo les hable en español porque algunas personas no me entenderían porque no lo hablan. Mi ombeayiüts es mi ombeayiüts por eso me gusta hablarlo. (A1, comunicación personal, 24 septiembre 2018)

También el alumnado de SDo y SFo manifiestan el interés que tienen en aprender la lengua en la escuela, ya que son conscientes de que en la comunidad hay casi nulas posibilidades de hacerlo, por ello manifiestan una apertura y actitud positiva hacia la lengua, como menciona un alumno de SFo:

Pienso que es bonito, que deberíamos de estudiarlo. Pienso también que es igual de importante que el español o el inglés, porque también se puede escribir y hablar por eso yo quisiera hablarlo, me gustaría estudiarlo muy bien, aunque es difícil el umbeyats pero me gusta mucho. Mi hermano que está en México me dice que aprenda la lengua que después me va a ayudar mucho si quiero seguir estudiando. Porque si ya no se habla, se nos va a olvidar. También mi papá me dice que es bueno hablar el huave, que, si me enseñan mis maestros que los aproveche y aprenda...Pero como los maestros tampoco hablan entonces hemos aprendido poco y ya es mi último año y me iré a la secundaria sin saber mucho. (A7, comunicación personal, 26 septiembre 2018 ).

La percepción de la niña de SMa y niño de SFo es contrastante, a pesar de que ambas comunidades presentan tendencias fuertes de desplazamiento de la lengua. Para la primera, el ombeayiüts es una lengua que ya no tiene ningún sentido ni significado, mientras que, para el segundo, aunque es consciente de que ya no se habla en su comunidad, representa parte de su identidad y manifiesta un interés por aprenderla en la escuela.

En el horario de clase del profesorado, excepto en SMa, se pudo observar, que la lengua aparece como asignatura y le asignan un horario para su enseñanza, aunque esto se limita a una o dos horas a la semana. En tanto que, desde el currículo nacional la distribución de horas no prioriza la situación de las lenguas indígenas.

TABLA 6

Distribución de horas semanales. Educación primaria

\begin{tabular}{|c|c|c|c|}
\hline \multirow{2}{*}{$\begin{array}{l}\text { Espacio } \\
\text { curricular }\end{array}$} & \multicolumn{3}{|c|}{ Grados/hrs semanales } \\
\hline & $1^{\circ}$ y $2^{\circ}$ & $3^{\circ}$ & $4^{\circ}-6^{\circ}$ \\
\hline Matemáticas & 5 & 5 & 5 \\
\hline Lengua materna & 8 & 5 & 5 \\
\hline Característica & $\begin{array}{l}\text { En educación } \\
\text { indigena, las ocho } \\
\text { horas de Lengua } \\
\text { Materna se dividen } \\
\text { en cinco horas para } \\
\text { Lengua Materna y } \\
\text { tres horas para } \\
\text { Segunda Lengua }\end{array}$ & $\begin{array}{l}\text { En educación } \\
\text { indígena, las cinco } \\
\text { horas de Lengua } \\
\text { Materna se dividen } \\
\text { en tres horas para } \\
\text { Lengua Materna y } \\
\text { dos horas para } \\
\text { Segunda Lengua }\end{array}$ & $\begin{array}{l}\text { En educación } \\
\text { indígena, las cinco } \\
\text { horas de Lengua } \\
\text { Materna se dividen } \\
\text { en } 2.5 \text { horas para } \\
\text { Lengua Materna y } \\
2.5 \text { horas para } \\
\text { Segunda Lengua }\end{array}$ \\
\hline
\end{tabular}

Fuente: Elaboración propia con base en datos de la Secretaría de Educación Pública [SEP], 2017.

Así, desde las disposiciones oficiales está estipulado que las matemáticas es el espacio curricular con mayor carga horaria, pero también, desde el 2017 la SEP tiene contemplado en los Programas de Estudio el apartado para lengua materna (tanto como L1 o L2) como muestra la Tabla 6, lo que refleja la continuación de una fragmentación de desarrollo lingüístico en la enseñanza, es decir, por un lado, las horas propuestas son muy limitadas para el proceso de una enseñanza integral de lenguas (Idiazabal, Manterola y Díaz, 2015; Ruiz, 2012; Pérez, Apraiz y Pérez, 2001) y por el otro, en la práctica educativa el profesorado no enseña la lengua por circunstancias multifactoriales como los que se ha anunciado y esto lleva a que en la mayoría de las escuelas 
indígenas exista una castellanización directa, pues no se considera ni la cultura indígena ni los estilos de aprendizaje de la población (Schmelkes, 2013).

Por ello, el profesorado aborda la lengua sin metodología específica y afirman “el tiempo que pueden”. Esto es confirmado por uno de las y los alumnos entrevistados:

El maestro si nos enseña el umbeyajts, lo tenemos en nuestro horario de clase. A veces nos enseña una o dos horas a la semana, pero también hay ocasiones o semana en que no lo trabajamos porque no nos da tiempo. Pero, aunque sea un poquito, si nos enseñan y hemos aprendido algunas palabras. (A4, comunicación personal, 14 septiembre 2018)

Otra realidad es que, aunque el espacio curricular de lengua materna se encuentre en las disposiciones oficiales, no todo el profesorado la trabaja. Como resaltan el alumnado de SDO y SFo:

\begin{abstract}
Algunos maestros nos han enseñado otros no, cuando hay algún concurso entonces nos dejan aprender alguna poesía o canto como el himno nacional, eso lo hemos aprendido en cuarto grado, pero solo nos dejan aprenderlo de memoria y luego repetirlo. (A4, comunicación personal, 24 septiembre 2018). Muy poco, las palabras que mencioné hace rato los aprendí en segundo grado... En tercero, cuarto y quinto nada, no nos enseñaron nada porque los profesores de aquí dicen que no saben hablar la lengua que por eso no nos enseñan, siempre nos comunicamos en español... (A7, comunicación personal, 26 septiembre 2018)
\end{abstract}

La realidad sociolingüística de las escuelas hace que la enseñanza de la lengua necesite de procedimientos diferenciados, dado que mientras para algunas el ombeayiüts es la L1 para otras es la L2 o incluso en SDo, cuenta con los dos perfiles. La situación de heterogeneidad de perfiles lingüísticos en el alumnado de las cuatro comunidades se ve complejizada por la problemática declarada por el profesorado tanto de formación para la enseñanza de la lengua, como de programas y materiales, agregando la cuestión de la escritura y de las variantes.

En SFo, el director declara que integrantes de la planta docente, a pesar de no hablar el ombeayiüts, investigan con las personas hablantes de la comunidad. Pero dichos esfuerzos están marcados por una concepción de la lengua como léxico y de la enseñanza como memorización:

Pero sí, algunos profesores hacen el esfuerzo de enseñarles a los niños algunas palabras o frases, lo que ellos saben o lo investigan con los señores de la comunidad y eso, lo retoman en el aula. También se promueve encuentros o concursos de cantos y poesías en la lengua, y los niños se motivan en aprender o memorizar palabras en la lengua (P1, comunicación personal, 26 septiembre 2018).

Esta forma de enseñanza es confirmada con la opinión de una alumna de SFo:

En quinto grado la profesora Berenice me dio una poesía en huave para que me lo aprendiera, y lo declamé el día de las madres. Me gustó mucho y me lo aprendí rápido, solo que no sabía que significaba algunas palabras, aunque la maestra me lo dio tanto en umbeyats como en español. (A8, comunicación personal, 26 septiembre 2018)

A partir de su experiencia de enseñanza, el profesorado se da cuenta de que debe haber una diferencia de enseñanza según el perfil lingüístico del alumnado. Como comenta una de las entrevistadas de SMo:

Trabajo en todo momento a partir del ombeayiüts porque es la primera lengua de los niños, es lo que hablan ellos. Les hablaba en español en ciertos momentos, muy poco porque no le entienden, por eso a fuerza uno les tenía que hablar en ombeayiüts, esto para el caso de los niños de primero y segundo grado. Cuando estuve en cuarto, ya retomaba las dos lenguas, casi mitad y mitad, porque los niños ya hablan un poco el español por el grado en donde están... (P7, comunicación personal, 08 noviembre 2018)

El profesorado realiza intentos de abordar la lengua en el aula, de acuerdo a sus propias formas de enseñanza y a su formación docente y académica. Pero sigue habiendo un vacío en la presencia de la lengua en cada una de las aulas del contexto ikoots al igual que en otros escenarios del estado y país. 


\section{REFLEXiones FinALES}

La presencia de la lengua indígena en las aulas ha estado marcada entre el mito y la realidad. En diversos contextos las lenguas indígenas están siendo sometidas por el español, dejándolas únicamente como puente o inexistentes en el espacio escolar. En el caso de la lengua ombeayiüts, su presencia y uso en el contexto escolar está enmarcada por factores como: el profesorado y alumnado ya no hablan la lengua, el desconocimiento sobre la escritura y metodologías de enseñanza y, sobre todo, el proceso de desplazamiento lingüístico. Este último, es uno de los elementos que más ha influido para el uso de la lengua en la escuela como es el caso de SFo y SMa y en la misma tendencia se encuentra SDo.

Otro de los factores que el profesorado señala es la incertidumbre para trabajar la lengua indígena, sea esta como primera o segunda lengua debido al desconocimiento de métodos y metodologías para la enseñanza. Esta realidad ha puesto como lengua de enseñanza al español en tres de las cuatro comunidades: SFo, SMa y SDo, solamente en SMo, el ombeayiüts se mantiene como medio comunicación, aunque como enseñanza es limitada. Dicha situación, pone también de relieve que, el desarrollo de una educación bilingüe (ombeayiütsespañol o viceversa) no se da tanto en escuelas de educación indígena como en la general, a pesar de que esta última está inmersa en un contexto ikoots.

\section{REFERENCIAS}

Barriga, R. (2018). De Babel a Pentecostés. Politicas lingüisticas y lenguas indigenas, entre historias, discursos, paradojas $y$ testimonios. México: SEP.

Bensasson, L. (2010). El Nábuatl en Morelos. Propuestas para la elaboración de métodos y materiales audiovisuales para la lengua Nábuatl (Tesis de Doctorado). Centro de Investigación y Docencia en Humanidades del Estado de Morelos, Morelos, México. Recuperado de https://catedracarlosmontemayor.files.wordpress.com/2020/03/te sis-laura-bensasson-2010.pdf

Bertely, M. (2000). Conociendo nuestras escuelas. Un acercamiento etnográfico a la cultura escolar. México: Paidós.

Brenner, M. (2006). Interviewing in Educational Research. En J. Green, G. Camilli y P. Elmore. (Eds.) Handbook of Complementary Methods in Education Research. (pp. 357-370). Mahwah (NJ): Lawrence Erlbaum Associates.

Brice, S. (1986). La politica del lenguaje en México: de la colonia a la nación. México: Instituto Nacional Indigenista.

Czarny, G. (2008). Pasar por la escuela. Indígenas y procesos de escolaridad en la Ciudad de México. México: UPN.

Dirección General de Educación Indígena [DGEI]. (2004). Políticas y fundamentos de la Educación Intercultural Bilingüe en México. México: SEP.

Fondo de las Naciones Unidas para la Infancia [UNICEF]. (2018). Informe anual. México 2018. México: UNICEF. Recuperado de https://uni.cf/2OS6fGm

García, P. (2017). Pueblos indígenas de México en el siglo XXI. Huave. México: CDI

Gasché, J. (2008). Niños, maestros, comuneros y escritos antropológicos como fuentes de contenidos indígenas escolares y la actividad como punto de partida de los procesos pedagógicos interculturales: Un modelo sintáctico de cultura. En M. Bertely, J. Gasché y R. Podestá. (Eds.) Educando en la diversidad. Investigaciones y experiencias educativas interculturales y bilingües. (pp. 279-365). Quito, Ecuador: Abya-Yala.

González, E. (2013). Apropiación étnica de la escuela entre los pueblos mixes de Oaxaca. La educación comunitaria e intercultural vista desde sus jóvenes. Perfiles Educativos, 35(141), 65-83. https://doi.org/10.22201/iisue.244 86167e.2013.141.40524

Hamel, R. y Muñoz, H. (1981). Bilingüismo, educación indígena y conciencia lingüística en comunidades otomíes del Valle del Mezquital, México". Estudios filológicos, 16, 127-162. Recuperado de http://hamel.com.mx/Archivos-Publicaciones/1981\%20bilinguismo\%20educacion\%20indigena\%20y\%20 conciencia\%20linguistica\%20en\%20comunidades\%20otomies\%20del\%20Valle\%20del\%20Mezqu.pdf 
Idiazabal, I., Manterola, I., y Diaz, L. (2015). Objetivos y recursos didácticos para la educación plurilingüe. En I. García-Azcoaga e I. Idiazabal. (Eds.).Para una ingeniería didáctica de la educación plurilingüe. (pp. 39-59) Bilbao: Universidad del País Vasco/Euskal Herriko Unibertsitatea.

Instituto Nacional de Estadística y Geografía [INEGI]. (2000). XII censo general de población y vivienda. México: INEGI. Recuperado de https://www.inegi.org.mx/programas/ccpv/2000/

Instituto Nacional de Estadística y Geografía [INEGI]. (2005). II conteo de población y vivienda. México: INEGI. Recuperado de https://www.inegi.org.mx/programas/ccpv/2005/

Instituto Nacional de Estadística y Geografía [INEGI]. (2010). Censo de población y vivienda. México: INEGI. Recuperado de https://www.inegi.org.mx/programas/ccpv/2010/

Instituto Nacional de Estadística y Geografía [INEGI]. (2015). Encuesta intercensal. México: INEGI. Recuperado de https://www.inegi.org.mx/programas/intercensal/2015/

Instituto Nacional para la Evaluación de la Educación [INEE] (2017). Directrices para mejorar la atención educativa de niñas, niños y adolescentes indígenas. México: INEE.

Martín, A. y, Escalante, C. (2017) Prácticas educativas, "el problema de la lengua" y usos indígenas de la lectura y la escritura en la historiografía de la educación del siglo XIX en México. Revista Mexicana de Investigación Educativa, 22(73), 347-366.

Meyer, L. y, Hamel, R. (2009). Enseñanza de la lengua materna y segunda lengua. Memoria del Segundo Congreso Nacional de Educación Indigena e Intercultural, 89-101.

Montero, G. (2012). El ombyayiüts en el aula: el caso de la Escuela Primaria Bilingüe "Moisés Sáenz" de San Mateo del Mar, Oax. México: UPN.

Muñoz, H. (2011). Reflexividad sociolingüistica de hablantes indígenas: Concepciones y cambio. México: UAM-I/ Ediciones del Lirio.

Pérez, T., Apraiz, M., y Pérez, M. (2001). El tratamiento integrado de las lenguas: los proyectos de comunicación. En U. Ruiz. (Coord.). Lengua castellana y literatura: Investigación, innovación y buenas prácticas. (pp. 151-173). Barcelona: Graó.

Rockwell, E., y González, E. (2012). Anthropological research on educational processes in México. En K. AndersonLevitt (Eds.). Mapping Anthropologies of Education. A Global Guide to Ethnographic Studies of Learning and Schooling. (pp. 107-138). Oxford, New York: Bergham Books.

Rockwell, E. (2009). La experiencia etnográfica. Historia y cultura en los procesos educativos. Buenos Aires: Paidós.

Ruiz, U. (2012). El plurilingüismo visto desde los documentos europeos: una mirada crítica. Textos de Didáctica de la Lengua y la Literatura, 60, 65-76. Recuperado de http://www.karrikiri.eus/sites/default/files/URIRUIZ.pdf

Saldívar, A. (2006). Técnicas y dinámicas para la educación intercultural. México: ECOSUR, Casa de la Ciencia, Instituto Austriaco de Cooperación Norte Sur.

Sandoval, E. (2004). Interculturalidad, una mirada desde abajo. Las relaciones interculturales de una comunidad indígena de Oaxaca, México. En S. Corona y R. Barriga (Coords.) Educación indígena. En torno a la interculturalidad (pp.57-65). México: Universidad de Guadalajara, Universidad Autónoma MetropolitanaXochimilco.

Sartorello, S. (2009). Una perspectiva crítica sobre interculturalidad y educación intercultural bilingüe: el caso de la Unión de Maestros de la Nueva Educación para México (UNEM) y educadores independientes en Chiapas. Revista Latinoamericana de Educación Inclusiva. 3(2), 77-90.

Schmelkes, S. (2013). Educación para un México intercultural. Revista Electrónica Sinéctica. 40, 1-12. Recuperado de http://www.redalyc.org/articulo.oa?id=99827467007

Secretaría de Educación Pública [SEP]. (2017). Plan y programas de estudio, orientaciones didácticas y sugerencias de evaluación. México: SEP.

Secretaría de Educación Pública [SEP]. (2015). Marco Curricular de la Educación Inicial Indígena y de la Población Migrante. Fundamentación normativa e Historia de la Educación Primaria Indígena. México: SEP. 
Serra, C. (2004). Etnografía escolar, etnografía de la educación. Revista de Educación, 334, 165-176. Recuperado de http://www.educacionyfp.gob.es/revista-de-educacion/en/numeros-revista-educacion/numeros-anteriores /2004/re334/re334-11.html

Sistema de Información de Tendencias Educativas en América Latina [SITEAL] (2018). México. La estructura del sistema educativo mexicano. Recuperado de https://www.siteal.iiep.unesco.org/sites/default/files/sit_accion_fi les/siteal_mexico_0101.pdf

Skutnabb, T. y Dunbar, R. (2010). Indigenous children's education as linguistic genocide and a crime against humanity? A global view. Galdu Čala, Journal of Indigenous Peoples Rights, 1, 1-128. Recuperado de https://w ww.afn.ca/uploads/files/education2/indigenouschildrenseducation.pdf

Staples, A. (2011). Una educación en desventaja. El México indígena (1821-1854). En L Alvarado y R. Ríos (Coords.), Grupos marginados de la educación (siglos XIX y XX). (pp.335-372). Ciudad de México: IISUE-UNAM/Bonilla Artigas Eds.

Tallè, C. (2015). La gramática de la identidad. La escuela bilingüe, los maestros y el "rescate" de la identidad en San Mateo del Mar (Oaxaca, México). ANUAC..(2), 157-188. https://doi.org/10.7340/anuac2239-625X-1982

Tubino, F. (2005). La interculturalidad crítica como proyecto ético-político. En Organización de Agustinos de Latinoamérica. Encuentro continental de educadores agustinos. Lima, Perú. Recuperado de https://oala.villanov a.edu/congresos/educacion/lima-ponen-02.html

Walsh, C. (2009). Interculturalidad, Estado, Sociedad: Luchas .de)coloniales de nuestra época. Quito: Universidad Andina Simón Bolívar; Abya Yala.

Zolla, C. y Zolla, E. (2004). Los pueblos indigenas de México. 100 Preguntas. México: UNAM.

\section{Notas}

[1] La estructura del Sistema Educativo Mexicano en cuanto a Educación Básica se refiere: la educación preescolar se ofrece en tres modalidades: general, indígena y cursos comunitarios, mientras que la primaria, se ofrece en tres servicios: general, indígena y cursos comunitarios (SITEAL, 2018).

[2] La cultura ikoots, comúnmente denominada como huave, está ubicada en las inmediaciones de la laguna superior e inferior del istmo de Tehuantepec. La palabra ikoots nosotros es una autodefinición por los propios habitantes, con cierta variación de acuerdo a la comunidad de procedencia: ikoots para SMo, konajts para SFo e ikojts para SDo y SMa. Son hablantes de la lengua ombeayiüts que significa nuestra boca, nuestra lengua, nuestra voz, al igual que las denominaciones de la cultura, el patronímico para referirse a la lengua también presenta variaciones sin cambio de significado: ombeayiüts para SMo, SDo y SFo la denominan umbeyajts y SMa umbeyujts. Para este trabajo se ha utilizado mayormente la variante de SMo, sin ninguna pretensión de imposición de una variante sobre otra, sino únicamente como referencia.

[3] El fenómeno de la desubicación lingüística es muy común entre el magisterio indígena de Oaxaca y de México. En cuanto a lenguas indígenas se refiere "es frecuente que el personal que habla una determinada lengua no necesariamente esté ubicado en una localidad donde ésta se hable, ello se debe a diversas situaciones (de carácter administrativo, sindicales y personales, entre otras)" (SEP, 2015, pp. 25-26).

[4] La entrevista tanto para el profesorado como para el alumnado, se organizó en apartados tendientes para conocer su experiencia académica y social con la lengua indígena.

[5] Se ha colocado únicamente una referencia para identificar a cada uno del profesorado entrevistado en la muestra, esto con motivo de guardar el anonimato. Solamente aparecen los datos reales de algunos padres y madres de familia que dieron su aprobación.

[6] Se ha respetado el anonimato del alumnado, por ello, se utilizan únicamente referencias.

[7] Las escuelas primarias tanto del medio indígena como generales se organizan de acuerdo al número de docentes que laboran en ella. Las clasificaciones son: organización completa (tiene los 6 grados en primaria y los tres niveles en preescolar atendidos cada grado/nivel por una persona docente), unitaria (una sola persona docente atiende diversos grados o niveles), bidocente (dos personas docentes atienden los grados o niveles existentes en la escuela), tridocente (tres docentes atienden los grados o niveles de la escuela), tetradocente (cuatro docentes atienden los seis grados o los tres niveles) y pentadocente (cinco docentes trabajan en la escuela).

[8] Término con el que se denomina a las personas ajenas a la cultura ikoots

\section{BY-NC-ND}

